\title{
La gerencia venezolana. Una perspectiva desde la complejidad
}

Venezuelan management. A perspective from social representations

Le management au Venezuela. Une perspective à partir des représentations sociales

Leslie Borjas de Xena lborjas@unimet.edu.ve Universidad Metropolitana, Caracas - Venezuela.

Posdoctorado en Ciencias de la Educación (UNESR, 2011)

Doctorado en Ciencias de la Educación (UNESR, 2007) Magister Scientarum en Desarrollo Organizacional (UCAB, 2001). Consultora en organizaciones públicas y privadas. Adscrita a la línea de

investigación "La gerencia venezolana desde la complejidad y aprendizaje organizacional”.

Dilia Monasterio de Márquez ailidad@cantv.net Universidad Nacional Experimental Politécnica de la Fuerza Armada Bolivariana, Caracas - Venezuela.

Posdoctorado en Ciencias Gerenciales (2007),

Doctorado en Ciencias

Económicas y Administrativas, USM (2004).

Magíster Scientarum en Gerencia de Personal. Instituto

Universitario de las Fuerzas Armadas, 1996.

Licenciada en Educación. Mención Administración, UCV, 1991.

Artículo de investigación científica y tecnológica Según Clasificación Colciencias

Fecha de recepción: $12 / 03 / 2012$

Fecha de corrección: $02 / 08 / 2012$

Fecha de aprobación: $05 / 12 / 2012$

\section{Resumen}

El objetivo de esta investigación es interpretar las representaciones sociales (RS) sobre la gerencia venezolana en organizaciones de naturaleza compleja, considerando su comprensión y acción a través de las prácticas administrativas, modelos organizacionales, emociones, expectativas $y$ actitudes que conforman la praxis gerencial. El enfoque teórico sigue los modelos de Moscovici, Jodelet y Abric. Morín señala que el pensamiento complejo es dialógico; esta corriente no objeta los datos de la experiencia, así como tampoco la validez de las leyes que rigen la naturaleza y la lógica que subyace a estas ciencias. En la investigación se asumió la complementariedad de métodos, mediante el uso de la estrategia de complementación (Bericat, 1998), se recurrió a métodos circunscritos al paradigma interpretativo, así como a los de carácter interpretativo. Los datos fueron obtenidos por la metodología de análisis de Spink. Las evidencias demuestran la presencia de dos lógicas en la gerencia venezolana donde la realidad entre el objeto y el sujeto observador se inscribe en un nuevo enfoque de realidades, complejidades y variedades, surgiendo las organizaciones que transitan entre los principios de incertidumbre y complementariedad en la cotidianidad.

Palabras clave: representación social, gerencia, complementariedad, complejidad. 


\section{Venezuelan management. A perspective from social representations}

La gerencia venezolana. Una perspectiva desde las representaciones sociales

Le management au Venezuela. Une perspective à partir des représentations sociales

\section{Abstract}

The object of this research was to interpret the Social Representations (SR) present in Venezuelan Management in organizations of complex nature considering their comprehension and action through administrative practices, organizational models, emotions, expectations, and attitudes, which form the general praxis. The theoretical approach follows the models by Moscovici, Jodelet, and Abric. Morin points out that complex thought is dialogical; this trend does not object to the data of experience nor the validity of the laws of nature and the logic behind these sciences. In the research, the complexity of methods was assumed by using complementation (Bericat, 1988), resorting to methods circumscribed to the interpretative paradigm. Data obtained were handled via Spink's analysis methodology. The outcomes show the presence of two logics in Venezuelan management where the reality between the object and the observer subject is inscribed in a new approach of realities, complexities, and varieties, emerging organizations which move between the principles of uncertainty and complexity in the everydayness.

Keywords: social representation, management, complexity.
Le management au Venezuela. Une perspective à partir des représentations sociales

La gerencia venezolana. Una perspectiva desde las representaciones sociales

Venezuelan management. A perspective from social representations

\section{Résumée}

Lobjectif de cette recherche est celui d'interpréter les représentations sociales (RS) du management vénézuélien dans les organisations à nature complexe, tenant compte de leur compréhension et leur action à travers les pratiques administratives, les modèles organisationnels, les émotions, les expectatives et les attitudes qui conforment la praxis managériale. L'approche théorique suit les modèles de Moscovici, Jodelet et Abric. Morin signale que la pensée complexe est dialogique ; ce courant nóobjecte pas les données de l'expérience, non plus la validité des lois qui régent la nature et la logique qui est sous-jacente à ces sciences. Dans la recherche, on a assumé la complémentarité de méthodes, à partir de l'usage de la stratégie de complémentation (Bericat, 1998), on a fait appel à des méthodes circonscrites au paradigme interprétatif, ainsi qú à ceux à caractère interprétatif. Les données ont été obtenues par le biais de la méthodologie d'analyse de Spink. Les évidences démontrent la présence de deux logiques dans le management vénézuélien, où la réalité entre l'objet et le sujet observateur s'inscrit dans une nouvelle approche de réalités, complexités et variétés, surgissant les organisations qui transitent entre les principes $d^{\prime}$ incertitude et de complémentarité dans la quotidienneté.

Mots clef: représentation sociale, management, complémentarité, complexité. 


\section{La gerencia venezolana. Una perspectiva desde las representaciones sociales}

\section{Introducción}

Las organizaciones del siglo XXI, han cambiado su forma de gerenciar. Al respecto Drucker $(1999,2002)$ señala que necesitan otros enfoques diferentes a los presentes en la literatura tradicional para su conducción. En este sentido, Motta (2001, p. 158) expresa que: "Las organizaciones están caracterizadas por la aceleración tecnológica, la alta competitividad gerencial, la demanda mayor de servicios públicos y la democratización del trabajo", que introdujeron nuevos enfoques que permiten a la gerencia ser flexibles para someterse a las exigencias cada vez más cambiantes en los ambientes de incertidumbre, caracterizados por la presencia de cambios tecnológicos y socio-culturales.

La gerencia venezolana se encuentra permeada por los cambios políticos, económicos, sociales, culturales y tecnológicos que orientan en este momento la dinámica del Estado venezolano. Éstos obedecen a nuevas realidades que emergen de la propia sociedad venezolana, donde se contraponen diversas perspectivas. Por consiguiente, la gerencia de las instituciones de carácter público y privado deben repensar las representaciones de las prácticas, métodos, estrategias y procesos que se desarrollan en el ámbito organizacional, cambios que transfiguran la cultura de la organización y su desempeño.

El reto de la gerencia venezolana en el siglo XXI, es aceptar las nuevas ideas que emergen desde una visión planetaria para su comprensión; sólo el gerente que perciba la complejidad de las procesos organizativos alcanzará entender la concurrencia de la complementariedad y de las contradicciones que emergen en las organizaciones. Desde esta perspectiva, el estudio tiene como objetivo interpretar las representaciones sociales (RS) sobre la gerencia venezolana en organizaciones de naturaleza compleja, considerando su comprensión y acción a través de las prácticas administrativas, modelos organizacionales, emociones, expectativas y actitudes que conforman la praxis gerencial en el contexto latinoamericano.

En el escenario venezolano, hay que considerar las situaciones vividas en Venezuela en el siglo XX, en dos momentos económicos: uno entre 1950 y 1978, donde el país creció a tal punto, de tener las tasas de crecimiento más altas de Latinoamérica y una inflación descendiente. El otro momento económico, se puede denominar "desastroso", o, "nuevo efecto
Venezuela", caracterizado porque a partir de 1978 , el producto interno bruto (PIB), sigue una tendencia decreciente, debido a políticas económicas erradas y a la carencia de una plataforma institucional que garantice la transparencia a los inversionistas.

En este contexto, Romero (2004) en un estudio realizado sobre la gerencia venezolana en la gestión pública, señala que se deben considerar las barreras que actualmente restringen el desempeño gerencial y entre estos obstáculos destaca: baja autoestima, prácticas y estilos de trabajo, pocas capacidades para liderar los cambios, poco atractivo en la función pública y alta rotación de funcionarios.

Monasterio (2008) indica que la gerencia del sector público enfrenta los nuevos dilemas éticos que emergen en las turbulencias de esta década (2000 al 2010). Las evidencias existentes revelan que en la administración pública hay poca capacidad para disipar los problemas éticos concretos de la vida cotidiana y los que surgen en un tiempo dominado por la incertidumbre.

Obuchi, Abadi y Lira (2011, p. 193) realizan una investigación en 16 empresas venezolanas, estudiadas en el marco de las políticas públicas durante 2002 a 2009, y señalan que la "evidencia histórica y la investigación académica muestran que el control estatal de la producción y planificación central suelen tropezar con algunas dificultades que conducen a problemas típicos de ineficiencia y escasez, como los que caracterizan a la economía venezolana”.

En el contexto venezolano, es necesario retomar las apreciaciones de Hamel y Prahalad (1999, p. 41) quienes plantearon "que los altos directivos ya no controlan el futuro de sus empresas", porque gestionan en ambientes llenos de incertidumbres y alejados del equilibrio. Por consiguiente, en este trabajo, se asume la complejidad como perspectiva teórica para comprender la gerencia actual.

\section{Aspectos teóricos}

La complejidad, según Maldonado (2001) es una nueva forma específica y nueva de racionalidad. Esta lógica de la complejidad, implica una nueva cosmovisión que se revela en forma conjunta y sin barrera a los criterios como orden, determinismo, estabilidad, causalidad, linealidad o previsibilidad. Asumir la complejidad requiere 
que la gerencia tenga la capacidad de concebir las nociones de organización, desorganización, autoorganización y aceptar de manera complementaria la coexistencia de los elementos físicos, biológicos, culturales, sociales, psíquicos, intelectuales y espirituales, que son inherentes al ser humano.

En este orden de ideas, Morín (2007) refiere que los tres principios del pensamiento complejo son: el principio dialógico, recursivo y el hologramático. El principio dialógico se basa en la asociación compleja (complementaria, concurrente, antagonista) de instancias necesarias juntas para la existencia, el funcionamiento y el desarrollo de un fenómeno organizado; por otra parte, el principio recursivo se basa en que todo momento es, a la vez, producto y productor, causante y causado, siendo el producto productor de lo que lo produce, el efecto causante de lo que causa y, finalmente, el principio hologramático parte del supuesto que no sólo la parte está en el todo, por el contrario, el todo, en cierto modo, está en la parte.

Las organizaciones sociales admiten que se requiere superar el pensamiento lineal ante la necesaria combinación de posibilidades que persiguen una visión mucho más real de los sistemas humanos, propia del devenir irreversible y la estabilidad, como factores de evolución del sistema social (Ciurana, 2000). Bajo esta óptica, la complejidad se presenta como la oportunidad que tiene la gerencia de asumir ambas posiciones, motivo por el cual aparecen nuevos elementos a ser considerados dentro de los enfoques gerenciales.

Maldonado (2001, p. 169) sostiene que "el pensamiento complexus significa lo que está tejido en conjunto. El pensamiento complejo es un pensamiento que busca, al mismo tiempo, distinguir -pero sin desunir-y religar".

La complejidad se permite la construcción del conocimiento a partir de ligar y religar paradojas por la unidad de lo múltiple, así como por la multiplicidad de lo uno, a partir de lo cual, así Morín (2003, p. 73) expresa:

...la extrema diversidad no debe enmascarar la unidad, ni la unidad profunda enmascarar la diversidad: La diferencia oculta la unidad, pero la unidad oculta las diferencias. Hay que evitar que la unidad desaparezca cuando las diversidades aparecen, que las diversidades desaparezcan cuando la unidad aparezca...
La complejidad es una posición epistemológica que admite la existencia de diversas formas de pensar, por tanto, los saberes y conocimientos son organizados de acuerdo con los principios que rigen una determinada lógica. En el caso de estudio de la gerencia, esta posición epistemológica permite avanzar hacia la comprensión de la realidad gerencial venezolana, sin descartar, en el presente estudio la explicación de algunos hechos desde una racionalidad clásica, partiendo de la posición expuesta por Morín (ob. cit.) que el pensamiento complejo es un pensamiento dialógico, en esta corriente no se objeta los datos de la experiencia, así como tampoco la validez de las leyes que rigen la naturaleza y la lógica que subyace a estas ciencias.

Los fenómenos sociales se estudian a través de la RS, modelo interno que tiene por función conceptualizar "lo real" aparte del sentido común, en el que el contenido significa una forma particular de pensamiento, designan una forma específica de conocimiento "el saber social" (Moscovici, 1984). Por consiguiente, las RS son tipos de creencias paradigmáticas, organizaciones de creencias, organizaciones de conocimiento y lenguajes y se encuentran en distintas estructuras de conocimiento.

La concepción de Moscovici (2003) plantea cuatro (4) elementos constitutivos de la RS. La información, que se relaciona con lo que "yo sé". La imagen que se relaciona con lo que "veo". Las opiniones con lo que "creo". Las actitudes con lo que "siento", elementos que se toman como guía para el análisis de la información. Desde esta mirada, las RS se especifican de acuerdo con su contenido, al considerar que entre las dimensiones que la constituyen se encuentran la información y la actitud. $L a$ información expresa la solidez del conocimiento que el sujeto exterioriza de un objeto social; por otra parte, la actitud, revela la orientación normal, positiva o negativa, frente al objeto de la representación.

Las RS de acuerdo con los enfoques de Banchs (1996-2000), Jodelet (2001), Moscovici y Marková (2003) son el resultado de construcciones cognitivas y perceptivas de la realidad la cual transforma las acciones sociales en categorías simbólicas (valores, creencias e ideologías) que a su vez, son auto reguladoras de las cogniciones y acciones y que conforman tipos de creencias paradigmáticas, organizaciones de creencias, organizaciones de conocimiento y lenguajes, y se encuentran en distintas estructuras de conocimiento. La representación es una acción psicológica que posee una función simbólica, porque implícitamente contiene un significado y éste tiene 
que ver de manera directa con la situación del sujeto frente al mundo en que vive y con el que se relaciona.

Las RS son precisadas a partir de dos componentes, los contenidos y la organización de esos contenidos en relación con un sistema central y otro periférico. Abric (1976), identifica como objeto de estudio la estructura y organización de las representaciones sociales y como objetivo de estudio, el desarrollo de la Teoría del Núcleo Central de la Representación, centrando su interés en el proceso de objetivación.

Las RS están organizadas alrededor de un núcleo y de unos elementos periféricos. El núcleo central (principio generador) tiene dos funciones esenciales: Una función generadora mediante la cual los otros elementos de la representación adquieren o trasforman su significado y una función organizadora de las relaciones que asocia los elementos de la representación. El abordaje de las RS para Abric (1994) permite entender la dinámica de las interacciones sociales y aclarar los determinantes de las prácticas sociales, en razón que la representación, el discurso y la práctica se generan mutuamente.

\section{Metódica}

El enfoque onto-epistémico que se asumió para el abordaje de esta investigación, concibe al ser como objeto de conocimiento, el cual acepta la realidad de manera multireferencial y cambiante, donde las explicaciones son un producto social y humano. De esta manera, en la relación observador-observado, no se acepta el acceso a una realidad única independiente del observador, existen tantas realidades como modos de vivir. Desde esta perspectiva, la persona es un ser único de naturaleza multidimensional en el cual lo biológico, lo psicológico y lo social se conforman diferencialmente (Morin, 2006). En este sentido, se reconoce que el conocimiento se constituye a partir de las experiencias propias de cada persona y del entorno social (Jodelet, 2001).

Por consiguiente, el estudio se inscribe dentro de la tradición que subraya la interacción entre sujeto y objeto de conocimiento, donde la construcción de la realidad son los aspectos legítimos que permiten al investigador la comprensión de la problemática en estudio, que de acuerdo con Murcia (2008, p. 94) quien plantea que desde el principio de complementariedad el investigador decide acudir a la "teoría formal para agregar la comprensión de aquellos eventos que la sola observación no permite interpretar”. Así, Murcia (ob. cit., p. 86) señala que "un enfoque desde la complementariedad no consiste en yuxtaponer métodos, es construir desde la comprensión del problema más adecuado para aproximarnos coexistencialmente a una realidad que nos aparece de manera compleja”.

Bericat (1998, p. 106) agrega que la integración de métodos en los procesos de investigación, pueden estar referidos a tres estrategias diferentes: complementación, combinación y triangulación. Para efectos de este estudio las investigadoras eligieron la estrategia de complementación, con el propósito de obtener dos imágenes, una cualitativa y otra cuantitativa de las representaciones sociales de la gerencia venezolana.

A la luz del argumento anterior, se realizó un proceso investigativo desarrollado en dos etapas; la primera correspondió a una estrategia de carácter cualitativo, discursiva, no convencional, de carácter no rígido; la segunda se enmarcó en una estrategia de carácter cuantitativo, siendo este último el método secundario, por consiguiente la indagación en el campo se realizó en diferentes tiempos de aplicación de acuerdo con los supuestos de Morgan [1988, citado en Bericat (1998)].

En un primer momento, la investigación se enmarcó dentro del paradigma interpretativo, según la lógica inductiva, mediante un trabajo de campo. Las estrategias de investigación se circunscribieron al enfoque cualitativo como lo señalan Santos y Madureira (2001, p. 20). Este tipo de metodología se refiere, en su más amplio sentido, a "la investigación que produce datos descriptivos: las propias palabras de las personas, habladas o escritas y las conductas observables". Actos y palabras a ser interpretados por el investigador con la finalidad de co-construir y reconstruir la realidad. En la Figura 1, se representan las preguntas que originaron la concepción sobre las RS de la gerencia venezolana.

La muestra estuvo conformada por doce informantes clave, que tenían como características ser gerentes venezolanos y expertos en el tema de gerencia con publicaciones sobre el área. Por la naturaleza del estudio, se practicaron las entrevistas a profundidad durante los meses de diciembre de 2010 a junio de 2011, mediante la utilización de un sistema de grabación, para garantizar mayor captación de la información que luego fue cotejada con los informantes.

El procedimiento seguido fue al proceso de categorización. En la primera fase se identifica- 
ron las unidades de significados, para luego categorizarlas y asignarles códigos a las categorías. La segunda fase, estuvo referido a un plano conceptual, que consistió en describir e interpretar las categorías; durante este nivel las categorías encontradas se compararon, con la finalidad de encontrar similitudes, diferencias y vínculos posibles. Después de haber realizado el proceso de categorización y estructuración, emergieron los nodos conceptuales para establecer la formación de redes de significados, dando inicio al proceso de interpretación y se establecieron las relaciones previas entre categorías, con la finalidad de entender el fenómeno estudiado.

Figura 1. Representaciones sociales sobre la gerencia venezolana

\begin{tabular}{|c|c|}
\hline $\begin{array}{l}\text { ¿De qué manera la gerencia } \\
\text { en organizaciones de } \\
\text { naturaleza compleja } \\
\text { construye las } \\
\text { representaciones sociales } \\
\text { como modelo de praxis? }\end{array}$ & $\begin{array}{l}\text { Las representaciones } \\
\text { sociales emergen por las } \\
\text { condiciones en que son } \\
\text { pensadas y constituidas, } \\
\text { surgen en momentos de } \\
\text { crisis y conflictos. } \\
\text { Moscovici (1961) }\end{array}$ \\
\hline Interrogante & —¿Qué son? \\
\hline $\begin{array}{l}\text { Representaciones } \\
\text { sociales sobre la } \\
\text { gerencia venezolana }\end{array}$ & $\begin{array}{l}\text { Se trata de fenómenos } \\
\text { observados directamente } \\
\text { o reconstruidos por } \\
\text { trabajo científico. }\end{array}$ \\
\hline $\begin{array}{c}\text { Gerencia } \\
\text { venezolana }\end{array}$ & -¿Para qué? \\
\hline $\begin{array}{l}\text { * Asistematización } \\
\text { * Incertidumbre } \\
\text { * Crisis } \\
\text { * Cambios continuos } \\
\text { * Conflicto }\end{array}$ & $\begin{array}{l}\text { Determinar los } \\
\text { procesos de anclaje y } \\
\text { objetivación en el } \\
\text { colectivo }\end{array}$ \\
\hline
\end{tabular}

Fuente: Borjas, L. y Monasterio, D. (2012).

El análisis de las entrevistas permitió categorizar la información sobre las prácticas gerenciales que ejecutan los gerentes, éste se hizo por la metodología usada por Spink (1999) procedimiento apropiado para el estudio de las RS.

El momento de la investigación desde el paradigma cuantitativo, consideró intencionalmente a 38 personas con experiencia en el área de gerencia y estudiantes de postgrado con cargos gerenciales. La técnica fue la entrevista semiestructurada mediante un guión con preguntas dirigidas a conocer: ¿Qué saben los gerentes venezolanos de la concepción de gerencia? ¿Qué creen los gerentes venezolanos de la gerencia? ¿Qué hacen los gerentes venezolanos en sus prácticas gerenciales?
Las consideraciones expuestas en los acápites anteriores en cuanto a la estrategia de complementariedad seguida en esta investigación, se presentan los resultados en dos etapas; la primera, correspondió a una estrategia de carácter cualitativo, discursiva, no convencional de carácter no rígido; la segunda, se enmarcó en una estrategia de carácter cuantitativo.

\section{Resultados}

La RS es un producto colectivo o conocimiento compartido, que emerge de las interacciones y que a su vez constituye y modula la interacción misma. Desde esta perspectiva, los hallazgos encontrados dan cuenta que las RS surgen ancladas al factor cultural. Por consiguiente, las interpretaciones dadas a los hallazgos evidenciaron como núcleo central de la RS que la cultura de la gerencia venezolana promueve la cultura del clan, donde prevalece el mantenimiento interno y la afiliación.

Al hablar de cultura en el contexto de la gerencia venezolana, se hace referencia a la conceptualización de Borjas (2011, p. 13) como significados compartidos que caracterizan la praxis de los individuos de una organización y que guían sus conductas. Cada organización está conformada por singularidades pertinentes, por lo cual, es difícil entender una organización si no se estudia su cultura, su evolución y desarrollo; por consiguiente la cultura puede ser evaluada desde las acciones de sus integrantes.

Las RS emergentes están ancladas en el microuniverso de los sujetos que pertenecen a la gerencia venezolana, así emergen los siguientes elementos como forma de conocimiento de sentido común de la realidad y dan cuenta de las RS de la gerencia venezolana, encontrándose en este estudio los siguientes contenidos representacionales: la prevalencia de lo individual sobre lo colectivo, la necesidad de afecto y reconocimiento de las personas, y el poder imbricado a la autoridad, conceptos imbricados en un fondo cultural el cual representa el núcleo central (Figura 2).

Estas representaciones evidencian una gerencia tradicionalista fundamentada en una racionalidad instrumental o técnica, donde lo individual es producto de un enfoque utilitarista del ser. Asimismo, en las organizaciones venezolanas, la motivación de las personas sigue estando centrada en la necesidad de afiliación, convirtiéndose las emociones en un elemento determinante en el ámbito organizacional. Por otra parte, el principio jerárquico de la autoridad, clásico de las organizaciones lineales lleva a que la 
noción de autoridad recaiga sobre los jefes o directores, generándose estructuras de poder vinculadas con la autoridad.

Figura 2. Representaciones sociales de la gerencia venezolana

Representaciones sociales de la gerencia venezolana

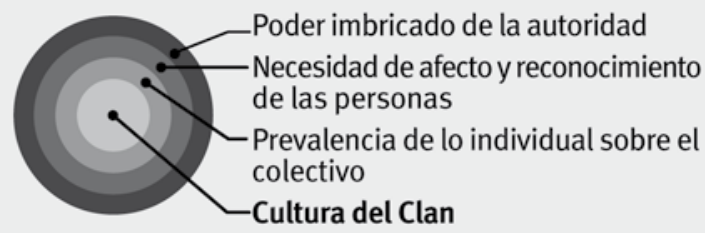

Construcción colectiva del objeto social

Fuente: Borjas, L. y Monasterio, D. (2012).

Estos hallazgos coinciden con los trabajos previos realizados por Romero García (2000) y Pietrosemoli (2006), donde los gerentes en sus discursos señalaron la necesidad de afiliación, prevalencia de lo individual, el control y el ejercicio de la autoridad; es evidente la ausencia de características que están presentes en las organi- zaciones del siglo XXI como innovación, transformación y tecnología. Otro componente del núcleo central de las RS son los "themata" (Moscovici, 1993) entendidas como formas de conocimientos irreflexivos o inconscientes y escasamente cuestionados, son temas persistentes con contenidos potenciales que proceden de la memoria colectiva y el lenguaje y a su vez participan en la producción de significados periféricos e imágenes asociadas con el núcleo central.

Los thematas expuestos (Figura 3), muestran los contenidos culturales compartidos con poderes generativos y normativos (o palabras más evocadas) de mayor recurrencia sobre la gerencia. Por consiguiente, se evidencia el conocimiento del sentido común que surge en la dinámica de la gerencia, donde conviven procesos técnicos administrativos (categorías tradicionales explicadas) y nuevas categorías (categoría emergentes) imbricadas al ser humano en la organización e interpretación que se hace con el propósito de comprender qué saben de la gerencia y cómo fundamentan lo que saben en el ejercicio de su praxis.

\section{Figura 3. Representaciones sociales sobre la gerencia venezolana}

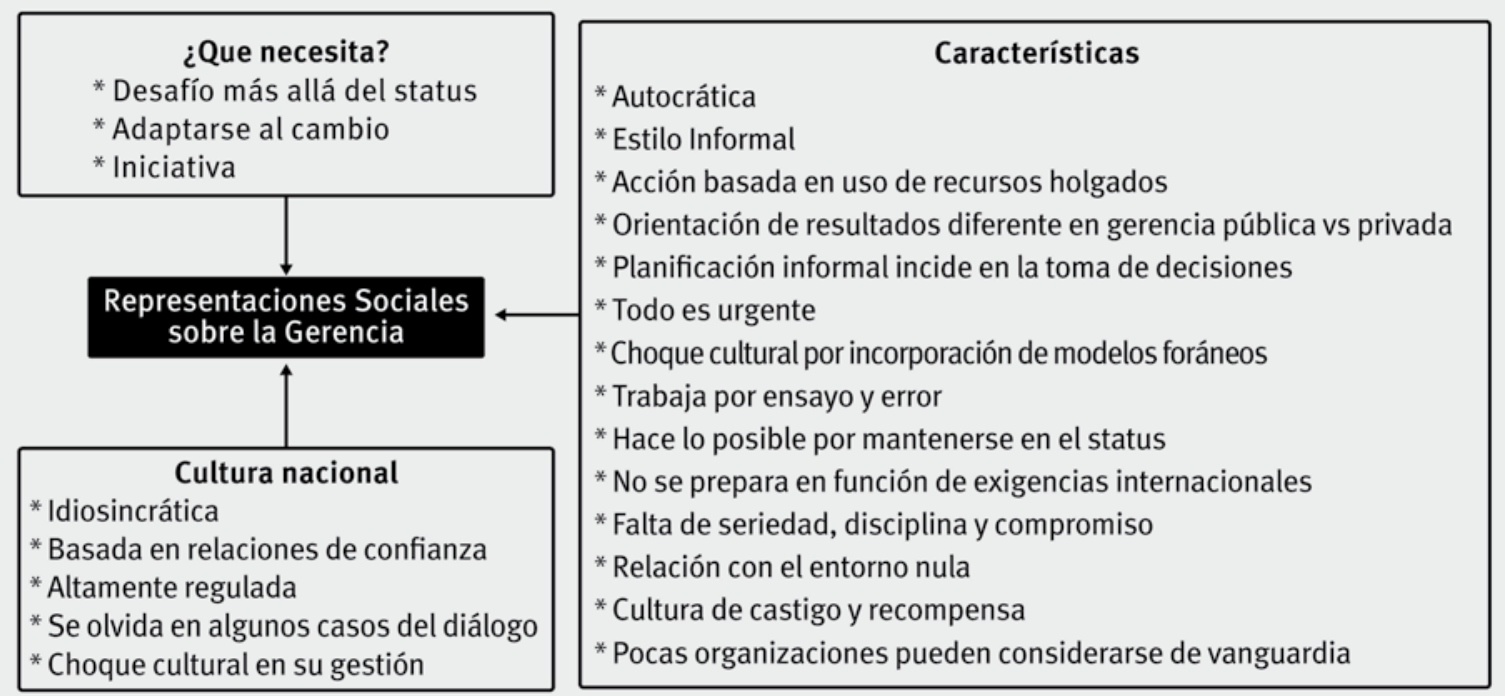

En la investigación, los contenidos configuran las RS de la gerencia, dando un sentido a la praxis gerencial venezolana, "no solo como un fenómeno cognitivo-conductual-emocional, sino como una vivencia compleja que se inserta en un marco socio cultural específico" (Arruda y de Alba, 2007, p. 290). Se trata, de una representación que tiene como núcleo central la cultura, específicamente la tipología conocida como "la cultura del clan" y está mediada por el componente emocional: "las emociones dominantes en un momento particular no son las que determinan la acción, sino el conjunto de necesidades y los efectos que la satisfacción de una de ellas pueda tener sobre intereses y metas" (Banchs, 1996, p. 119).

Las RS de la gerencia venezolana como sistemas cognitivos de los gerentes en su práctica cotidiana, reflejan la presencia de estereotipos, opiniones, creencias, valores, principios interpretativos orientadores de las prácticas gerenciales. 
En el segundo momento de la investigación, se recurrió a técnicas estadísticas descriptivas para obtener aspectos diferentes en cuanto a las representaciones sociales de la gerencia desde una perspectiva de la complementariedad. En este orden, fueron identificadas tres categorías: noción de la gerencia (¿Qué se sabe?), interpretación de la gerencia venezolana (¿Qué se cree?) y prácticas gerenciales (¿Qué se hace?).

\section{Noción de la gerencia (¿Qué se sabe?)}

Esta categoría refleja los conocimientos de los gerentes acerca del tema. Los resultados obtenidos señalan que $55 \%$, corresponden a la concepción de planificación, $24 \%$ lo relacionan con la función de coordinación y el restante $21 \%$ con la dimensión política.

Esta representación está relacionada con modelos gerenciales que reciben durante su formación educativa. Por consiguiente, los términos utilizados para describirla dan cuenta de las concepciones de planificación estratégica que cimenta la curricula en materia de gerencia y administración.

La función de coordinación, es el segundo saber reportado, conceptualizada por Cameron y Quinn (2005) como un tipo de cultura jerárquica, donde el líder ejerce los roles de coordinador, monitor y organizador. Este tipo de cultura está fundamentado en los supuestos de estabilidad y control, en el cumplimiento de las normas organizacionales, por cuanto los roles están establecidos a través de normas y regulaciones. Los líderes se enorgullecen de que son buenos coordinadores y organizadores, efectivamente cuidadosos. Se enfatiza el alto control y enfoque externo.

En el rol de "monitor" (Borjas, 2010) se espera que el directivo esté al día de todo lo que sucede en su unidad de trabajo y compruebe si las personas cumplen las normas y la unidad cumple su cuota, conozca todos los hechos y detalles y sea buen analista. En el rol de "coordinador", el directivo debe mantener la estructura y flujo del sistema. La persona que desempeña este rol debe ser digna de confianza y veraz. Sus rasgos conductuales incluyen diversas formas de facilitar el trabajo, por ejemplo, la programación, la organización y la coordinación del personal, la solución de situaciones de crisis; también es responsable de los temas tecnológicos, logísticos y de mantenimiento.
Otra categoría emergente fue la política (21\%) como RS de la gerencia. El concepto de política general de la empresa e instituciones como concepto integrador de la dirección, inicia el origen del paradigma de la estrategia y de una importante corriente del pensamiento, aplicable a la gerencia pública. Para Bédard (2003) el concepto de política general trata sobre las tres funciones de la empresa: política, estratégica y económica. Este concepto describe la responsabilidad específica de la dirección y tiene identidad como conceptualización de la política general en la gerencia pública y privada, la elección de los objetivos, el desarrollo de la identidad, la evaluación de las acciones, la movilización de los recursos y la definición de los estándares.

\section{Creencias sobre la gerencia venezolana (¿Qué se cree?)}

Los resultados en cuanto al "qué se cree", la gerencia venezolana estuvo caracterizada por la improvisación (29\%), el desorden (26\%), el conservadurismo $(21 \%)$, el cortoplacismo (13\%) y el autoritarismo (11\%).

\section{Prácticas gerenciales. (¿Qué se hace?)}

Las RS sobre las prácticas gerenciales, están imbricadas a la forma "¿Qué se hace?" y el "Cómo se actúa" (actitud) en la gerencia venezolana. Esta dimensión es el elemento más afectivo de la representación, por ser la reacción emocional acerca de la práctica gerencial que acontece en ámbito de las organizaciones actualmente. Además, se construye a partir de la experiencia y permiten valorar o calificar los hechos.

En este sentido, Monasterios y González (2012) a los supuestos por Jodelet (1984), expresan que la noción de RS concierne a la forma en que los sujetos sociales aprenden los acontecimientos de la vida diaria, las características del medio ambiente y las informaciones presentes. De esta manera, las representaciones sociales constituyen un conocimiento estructurado con un carácter dinámico y funcional; es decir, las personas, dentro de la posición que ocupan en la estructura social, dentro de un grupo social de pertinencia y a partir de la interacción con otros, reciben una información, la redefinen y la anclan a un fondo común de conocimientos.

En la investigación, los gerentes consideran que las prácticas en las organizaciones están orienta- 
das hacia los resultados ( $45 \%$ ), la emocionalidad $(19 \%)$ y orientada a la innovación (16\%). Otras categorías que surgieron son centrada en la ética $(5 \%)$, centrada en la cultura (5\%), centrada en las personas (5\%) y centrada en los procesos (5\%).

La orientación de las prácticas gerenciales según la opinión del grupo estuvo orientada en alto porcentaje ( $45 \%$ ) al logro de los resultados, lo que demuestra la preeminencia de los modelos gerenciales centrados en resultados, donde prevalecen lógicas que tienden a gerenciar el contenido socioeconómico, centrándose en el "homo economicus" como centro de la acción gerencial de carácter mecanicista. Sin embargo, emergen nuevos elementos como la emoción, la innovación, la ética, categorías que conforman las acciones humanas y devela otros contenidos que reflejan tímidamente una gerencia con tendencia a la comprensión del "homus social" en las instituciones venezolanas.

En este contexto, las representaciones sociales según Monasterio y González (2012) sobre la gerencia como forma de conocimiento espontáneo, ingenuo e intuitivo difiere al conocimiento científico, son conocimientos que se instituyen a partir de las experiencias, informaciones, conocimientos y modelos de pensamiento recibidos y transmitidos a través de la tradición, el saber, la experiencia y el trayecto recorrido por los gerentes que formaron parte del estudio.

\section{Conclusiones}

La gerencia como sistema social está impregnada por las transformaciones y cambios propios de la organización, donde tradicionalmente son gestionadas dentro de una racionalidad mecanicista e instrumentalista y regidas por métodos convencionales de gestión.

En la gerencia venezolana, las RS constituyen una construcción sociocognitiva, caracterizada por la presencia de una disonancia entre la noción de gerencia del saber conocida y la praxis gerencial develada en el quehacer organizacional. De esta manera, conviven en el espacio corporativo las paradojas, valores opuestos unos a los otros, compartidos y no compartidos por el grupo gerencial.

Las autoras concluyen que de las organizaciones estudiadas en el contexto de la Venezuela actual existe la necesidad de repensar los modelos gerenciales que dan cuenta de la actuación de los gerentes e incorporar prácticas de carácter humano que permitan orientar la construcción de nuevos modelos centrados en las personas. Se reitera que el accionar gerencial debe trascender la lógica cosificante del modelo de organización prevaleciente hacia un modelo de organización participativo e incluyente, donde los seres humanos conviven y se esmeran por lograr un espacio digno con sus pares en la sociedad venezolana.

En el contexto actual, la gerencia venezolana enfrenta entre sus más importantes retos, apostar a nuevas prácticas que permitan alcanzar la transformación de las instituciones, exigencia que demanda la actual coyuntura socio-histórica que vive el país. Este desafío implica transitar por una noción de gerencia que incluya las estrategias, el sistema de valores, lo estético, lo ético y lo político.

Los hallazgos evidencian que en la plenitud del siglo XXI, en el caso de la gerencia venezolana, existen prácticas gerenciales que permiten develar la racionalidad que impera en la cotidianidad y diferentes anclajes en las conversaciones de los actores acerca de "modas gerenciales" en sus prácticas que son antagónicas al desarrollo intrínseco de las organizaciones que conducen.

Los resultados de este estudio evidenciaron como núcleo central de la RS que "la cultura de la gerencia venezolana promueve la cultura del clan (Cameron y Quinn, 2011) donde prevalece el mantenimiento interno y la afiliación. Otras RS que emergieron fueron: prevalencia de lo individual sobre lo colectivo, necesidad de afecto y reconocimiento de las personas, poder imbricado a la autoridad.

El estudio de las RS sobre la gerencia venezolana parte del supuesto que la práctica gerencial está imbricada a la noción de organización, que per se es compleja; de esta forma se constituye como una fibra organizacional que emerge de las relaciones e interrelaciones entre las partes o eventos, permitiendo que éstas se consoliden y subsistan temporalmente dentro del sistema social. La investigación apuntala a reconocer la gerencia desde una perspectiva compleja, donde los elementos son productos de las acciones y relaciones que irrumpen en la cotidianidad de la gerencia venezolana.

Los supuestos generados en este estudio dan cuenta que las RS presentes en la gerencia venezolana son producto de una coyuntura socio-política que ha permeado la acción de la gerencia venezolana del siglo XXI. Por consiguiente, las acciones 
pendientes es construir un nuevo modelo de gestión que tenga como eje central lo humano, sus valores, actitudes y creencias, símbolos, historias, ritos, mitos, arquetipos, el lenguaje, el sentido y el espacio físico, así como lo racional de lo humano en una constante definición de lo organizacional de su sistema.

Las construcciones del conocimiento de la gerencia genera la necesidad de realizar nuevos estudios donde asuma la complejidad como un elemento de comprensión de la gerencia en el siglo XXI marcada por una resignificación de lo humano en las organizaciones.

El aporte de este trabajo es haber utilizado el enfoque complementario para intentar clarificar un fenómeno: la gerencia venezolana, caracterizada como un fenómeno complejo en organizaciones que viven impacto socio-cultural y que aún, están regidas por un proceso de transición entre la gerencia tradicional y las exigencias de las organizaciones caracterizadas en la sociedad del conocimiento.

\section{Referencias}

Abric, J. (1994). Les rèpresentations sociales. Aspects theoriques. En: Abric, J.C (Dir.). Practiques sociales et Rèpresentations. París, Francia: Presses Universitaires de France.

Arruda, A., De Alba, M. (2007). Espacios imaginarios y representaciones sociales. Aportes de desde Latinoamérica. Iztapalapa, México: Anthropos.

Banchs, M. (1996). El papel de la emoción en la construcción de representaciones sociales: invitación para una reflexión teórica. Papers on Social Rèpresentations, 5 (pp. 113-125).

Banchs, M. (2000). Aproximaciones procesuales y estructurales al estudio de las representaciones sociales. Papers on Social Rèpresentations, 9 (pp. 3.1-3.15).

Bédard, R. (2003). Los fundamentos del pensamiento administrativo: El rombo y las cuatros dimensiones filosóficas. Revista Ad-Minister, (3) (pp. 68-88).

Bericat, E. (1998). La integración de los métodos cuantitativos y cualitativos en la investigación social. Barcelona, España: Ediciones Ariel S.A.

Borjas, L. (2010). Cultura y liderazgo en una empresa de servicios venezolana. Anales de la Universidad Metropolitana, 10 (1) (pp. 139162).

Borjas, L. (2011). Cultura y Liderazgo basado en Aprendizaje Organizacional: Un enfoque desde la complementariedad. Saarbrücken, Alemania: Académica Española.

Cameron, K. \& Quinn, R. (1999). Diagnosing and Changing organizational cultura, base on the Competing values framework. New York, USA: Addison Wesley Publishing Company.

Cameron, K. \& Quinn, R. (2011). Diagnosing and Changing Organizational Culture: Based on the Competing Values Framework ( $3^{\mathrm{a}}$ ed.). San Francisco, USA: The Jossey-Bass Business \& Management Series.

Ceballos, R., González, C.H.; Parra, A.V. y Illiera, J.C. (2012). Guía de autores. Cuadernos de Administración 28 (47) (pp. 114-121).

Ciurana, E.R. (2000). El modelo organizacional, su Método. Valladolid, España:Valladolid.

Drucker, P. (1999). Gerencia organizacional. Ciudad de México, México: Mc Graw Hill.

Drucker, P. (2002). Los Desafíos de la gerencia para el siglo XXI. Bogotá, Colombia: Grupo Editorial Norma.

Drucker, P. (2002). La gerencia en la sociedad futura. Bogotá, Colombia: Editorial Norma.

Jodelet, D. [comp.] (2001). As representações sociais. Rio de Janeiro, Brasil: Universidade do Estado do Río de Janeiro.

Hamel, G. y Prahalad, C. (1999). Compitiendo por el futuro. Boston, USA: Harvard Bussiness Review.

Maldonado, C. (2001). Visiones sobre la Complejidad. Bogotá, Colombia: Kimpres Ltda.

Monasterio, D. y González, M. (2012). Una perspectiva del buen vivir, a propósito de la administración pública. Filosofías del buen vivir, del mal vivir y otros ensayos. Caracas, Venezuela: Editores Gregorio Valera, Gladys Madriz.

Monasterio, D. (2008) Una aproximación a la comprensión de la ética en la gerencia pública. Revista Ensayo y Error, 35 (pp. 25-47). 
Morín, E. (2007). Introducción al pensamiento complejo. Barcelona, España: Edit. Gedisa.

Morin, E. (2007). Introducción al pensamiento complejo ( $9^{a}$ reimpresión); Barcelona, España: Edit. Gedisa.

Morín, E. (2004). ¿Podemos reformar la administración pública? IX Congreso Internacional del CLAD sobre la Reforma del Estado y de la Administración Pública, Madrid, España, 3-5 noviembre. CLAD.

Morín, E. (2003). El método V: La humanidad de la humanidad. La identidad humana. Madrid, España: Ediciones Cátedra, Grupo Anaya.

Moscovici, S. y Castorina, J.A. (2003). Representaciones sociales. Problemas teóricos y conocimientos infantiles. Barcelona, España: Edit. Gedisa.

Moscovici, S. \& Georges, V. (2001). The concept of Themata. En: Duveen, G. (Ed.). Social representations. Explorations in social psychology. New York, USA: University Press.

Moscovid, S. y Marková, I. (2003). La presentación de las representaciones sociales: diálogo con Serge Moscovici. En: Castorina, J. (Comp.). Representaciones sociales. Problemas teóricos y conocimientos infantiles. Madrid, España: Editorial. Gedisa.

Moscovici, S. (1993). Introductory adrress to the international conference on social representations. Paper on Social Representations, 2 (3) (pp. 160-170).
Motta, P. (2001). Transformación organizacional. Bogotá, Colombia: Ediciones Uniandes, Facultad de Administración, Alfa-Omega.

Obuchi, R., Abadi, A. y Lira, B. y Obuchi, R. (2011). Gestión en rojo. Evaluación del desempeño de 16 empresas estatales y resultados generales del modelo socialista productivo. Caracas, Venezuela: Edicines IESA, Instituto de Educación Superior Caracas Venezuela.

Pietrosemoli de-Dikdan, L. (2006). ¿Por qué fortalecer el espíritu empresarial venezolano? Entorno-Empresarial.Com. Recuperado 30/07/2011 de: http://www.entorno-empresarial.com/?ed $=65 \&$ pag $=$ articulos $\&$ aid $=348$

Romero, G. (2005). Papel del gerente líder venezolano. Revista de Estudios Interdisciplinarios en Ciencias Sociales, 7 (1) (pp. 51-65).

Romero, J. (2004). Una epistemología para la administración pública venezolana. Caracas, Venezuela: Ediciones de la OPSU.

Romero-García, O. (2000). Espíritu empresarial: Una visión psicosocial. Recuperado 15/11/2010. de: http://www.analitica.com/va/ economia/organizacion/ 9473447.asp

Santos, A. y Madureira, J. (2001). Metodología das ciencias socias. Porto, Portugal: Editorial Edicões Afrontamento.

Spink, M.J. (1999). Prácticas discursivas e produccao de sentidos no cotidiano. Sao Paulo, Brasil: Cortéz. 